\title{
LA PREVENCIÓN DEL BURNOUT EN LAS ORGANIZACIONES: EL CHEQUEO PREVENTIVO DE LEITER Y MASLACH (2000)
}

\author{
SANTIAGO GASCÓN, MARGARITA OLMEDO, HÉCTOR CICCOTELLI \\ Universidad Nacional de Educación a Distancia (UNED)
}

(Aceptado en julio de 2002)

\begin{abstract}
El burnout, o síndrome de quemarse en el trabajo, ha alcanzado proporciones epidémicas en el mundo laboral. Dadas las graves consecuencias que este fenómeno tiene, tanto para la salud de los trabajadores, como para el buen funcionamiento de las organizaciones se han propuesto varias formas de tratamiento entre las que priman las estrategias individualizadas. Sin embargo, en los últimos años, se ha hecho evidente la necesidad de modificar las variables relacionadas con los estresores vinculados al puesto de trabajo. Los doctores Leiter y Maslach (2000) elaboraron un procedimiento para evaluar las áreas de riesgo de burnout en una organización e iniciar, con los datos resultantes, un programa de intervención encaminado a transformar las condiciones de la empresa y disminuir con ello la probabilidad de que aparezca el burnout. El objetivo del presente artículo es la presentación de dicho procedimiento y discutir sus características frente a otros tipos de intervención.
\end{abstract}

Palabras clave: Síndrome de quemarse en el trabajo, burnout, estrés laboral, salud, prevención, afrontamiento.

\section{Preventing burnout in organizations: \\ The preventing checkup of Leiter and Maslach (2000)}

Burnout is reaching epidemic proportions in the workplace. Several empirical studies of the effect of stress and burnout on health have shown serious consequences for workers and organizations. Until now, most of the intervention programs in reducing the effects of burnout were individual and focused on person's crisis. During the last years it has been more evident the need to transform variables related to the working conditions itself, specially in those workplaces where the risk of burnout were probably to occur. Leiter and Maslach (2000) developed a procedure to evaluate the risky areas of the organization and start, with the result of an evaluation and intervention program aimed to change those organizational conditions and to diminish the probability of burnout. The objective of this article is to present this intervention procedure and to discuss its characteristics in contrast to other ways of intervention.

Key words: Burnout, work stress, health, prevention, coping.

\section{ESTRÉS LABORAL Y BURNOUT}

El concepto de burnout fue introducido por Freudenberger (1974) para describir el estado de agotamiento físico y mental que observó entre voluntarios de una clínica de desintoxicación. Posteriormente, Maslach $(1976,1981)$ lo popularizó, refirién-

Correspondencia: Margarita Olmedo, Dpto. de Psicología de la Personalidad, Evaluación y Tratamiento Psicológicos, Facultad de Psicología, UNED, c/ Juan del Rosal 10 (Ciudad Universitaria), 28040 Madrid. Correo-e: molmedo@psi.uned.es. dose al desgaste profesional que sufren los trabajadores de los Servicios Humanos y lo definió como un síndrome cuyos síntomas son el cansancio emocional, la despersonalización y la falta de realización en el trabajo. Burke (1987) entiende que es un proceso de adaptación al estrés laboral crónico, cuando han fallado las estrategias de afrontamiento del sujeto. Es frecuente en profesionales de la salud, de la educación y, en general, en quienes trabajan en servicios humanos, pero en la actualidad se aplica a un amplio colectivo de profe- 
siones (Burke, 1987; Cherniss, 1980; GilMonte y Peiró, 1996; Harrison, 1983; Maslach y Jackson, 1982).

El burnout es uno de los problemas más graves que afectan al mundo laboral, con enormes consecuencias tanto personales como económicas. Puede afectar a la salud física y mental, dando lugar a alteraciones psicosomáticas (cardio-respiratorias, jaquecas, úlceras, insomnio, etc.), estados ansiosos, depresivos, alcoholismo, etc. (Bronsberg y Vestlund, 1997; Buceta y Bueno, 1995). Para la empresa supone un mayor índice de absentismo, rotaciones excesivas, incluso abandono del puesto por parte del trabajador, traduciéndose en deterioro del rendimiento y de la calidad (Maslach y Leiter, 1999). $\mathrm{El}$ estrés es la segunda causa de baja laboral en la Unión Europea, afectando anualmente a cuarenta millones de trabajadores y suponiendo para sus países miembros un coste de 20.000 millones de euros al año, entre gastos sanitarios, sin contar la pérdida de productividad. Es pues un problema que no sólo preocupa a las personas afectadas, sino también a los directivos y a los responsables de recursos humanos y de prevención de riesgos laborales. Por ello, cada vez es más común que las empresas promuevan programas de evaluación y tratamiento del estrés entre sus empleados.

Sería prácticamente imposible tratar de identificar todas las variables implicadas en el síndrome de quemarse por el trabajo, pero sí suelen agruparse en fuentes de estresores y en ciertas características personales que presentan mayor vulnerabilidad ante estos estímulos (Gil-Monte y Peiró, 1996). Un grupo de variables tienen que ver con las características organizacionales del puesto de trabajo, como el ambiente físico, la cultura de la empresa, las relaciones interpersonales, o el clima laboral (Jenkins y Calhoun, 1991; Mallet, 1991); otras proceden de factores externos a la empresa y relacionados con el trabajador, como los problemas familiares, o las dificultades económicas; y juegan también un papel importante las propias características del individuo (variables demográficas, rasgos de personalidad, etc.) a la hora de determinar la naturaleza y amplitud de los recursos biológicos y psicológicos que puede utilizar para responder a las demandas (Lázarus y Folkman, 1994). Éstas últimas suelen considerarse variables moduladoras o variables antecedentes del estrés (Buceta y Bueno, 1995; Gil-Monte y Peiro, 1996; Parkes, 1998). Varios autores han tratado de definir el proceso por el cual el estrés deviene en burnout. Para Cherniss (1980), se trata de un proceso de acomodación psicológica progresiva entre un trabajador estresado y un trabajo estresante, distinguiendo varias etapas: 1) una fase de estrés, caracterizada por un desajuste entre las demandas laborales y los recursos del trabajador; 2) una fase de agotamiento, al producirse de forma crónica respuestas de preocupación, tensión, ansiedad y fatiga; y 3) una última etapa de agotamiento defensivo, en la que se aprecian cambios en las actitudes y en la conducta, como la robotización y el cinismo. Por su parte, Leiter (1988) estableció la siguiente secuencia: cansancio emocional, fruto del desequilibrio entre demandas y recursos; despersonalización, en respuesta a la situación creada; y falta de realización personal, como resultado del choque entre las estrategias de afrontamiento y el compromiso que se exige (Manzano y Ramos, 1999). Algunos modelos explicativos ponen más énfasis en las variables de tipo personal (Harrison, 1983), otros en los procesos de interacción social (Buunk y Schaufeli, 1993) y otros en la importancia de las variables organizacionales (Golembiewski, 1982; Leiter y Maslach, 2000). Una única perspectiva no parece dar una explicación completa y satisfactoria sobre la etiología del síndrome, que pasa por integrar varia- 
bles recogidas en las tres perspectivas. La evidencia empírica de los modelos explicativos es muy limitada por razones conceptuales y metodológicas y son necesarios estudios de carácter longitudinal que den apoyo empírico a cualquiera de los modelos.

El diagnóstico y evaluación del burnout se ha venido realizando desde la observación clínica, entrevistas estructuradas, técnicas proyectivas y autoevaluación con escalas de estimación. Exceptuando las medidas de autoinforme, que recogen información de los cambios emocionales, cognitivos y conductuales, el resto de las técnicas han sido raramente empleadas en investigación. Algunos de estos instrumentos son: el Staff Burnout Scale for Health Professionals (SBS-HP; Jones, 1980), el Tedium Measure (TM; Pines, Aronson y Kafry, 1981). Maslach y Jackson (1981, 1996) elaboraron el Maslach Burnout Inventory (MBI) para medir el burnout como constructo multidimensional. La escala evalúa las tres dimensiones de cansancio emocional (CE), despersonalización (DP) y falta de realización (RP), por medio de tres subescalas. Por su sencillez, el MBI ha sido ampliamente utilizado y los estudios de validación han mostrado el contenido exclusivo y diferencial del síndrome de burnout respecto de otros conceptos psicopatológicos como el estrés, la fatiga, la insatisfacción laboral, la ansiedad o la depresión (Leiter y Schaufeli, 1996; Maslach y Jackson, 1981; Maslach, Jackson y Leiter, 1996; Shirom, 1989).

\section{TRATAMIENTO Y PREVENCIÓN DEL BURNOUT}

Como se ha apuntado, el síndrome de quemarse en el trabajo es un problema complejo y difícil de abordar, dada la cantidad de variables implicadas. El tipo de intervención predominante ha sido la individualizada y centrada en la persona en crisis (Pines y Aronson, 1988; Ramos, 1999) abordándose normalmente desde programas de entrenamiento para el manejo del estrés, que suelen agrupar distintas técnicas dirigidas a producir cambios en la persona. Existen programas específicos para tratar algún aspecto concreto del problema, pero son más comunes los que contemplan distintas variables relacionadas con el proceso de estrés y sus antecedentes, junto con entrenamiento en gestión del tiempo, en estrategias de afrontamiento y modificación de los estilos de vida (Olmedo y Santed, 1999). Sin duda esto es debido a que la visión generalizada ha sido la de que el individuo se quema por algún defecto en su carácter o en su conducta (Maslach, 1999; Maslach y Leiter, 1999), de modo que si el problema está en el individuo, la solución es que éste cambie. Como resultado de sus trabajos, Leiter y Maslach (Leiter y Maslach, 2000; Maslach y Leiter, 1999) creen que el burnout es fundamentalmente responsabilidad del medio social en el que se trabaja, cuya estructura y funcionamiento se forma por interacción entre las personas.

Lamentablemente, el síndrome de quemarse en el trabajo suele afrontarse cuando ya es un problema, suponiendo un alto coste en compensaciones, subsidios, bajas y pérdidas por errores laborales y deterioro de la calidad. Por ello, en los últimos años va siendo cada vez más normal que las empresas tomen la iniciativa de cara a la prevención, ya que son responsables del diseño y configuración del entorno laboral y han comprendido que es una buena inversión para el futuro. Pero, incluso desde un punto de vista preventivo se ha mantenido el énfasis en dotar a los trabajadores de estrategias para su afrontamiento, más que en cambiar las condiciones objetivas de la organización (Golembiewski, 1986).

Al hablar de prevención debemos distinguir entre los factores de riesgo y los 
mecanismos mediante los cuales estos factores de riesgo llegan a producir un trastorno determinado y que suelen incluir una secuencia compleja de interacción entre las características personales y el ambiente laboral. Por ello, hay dos formas de prevenir: reducir esos posibles factores de riesgo, e identificar los casos pre-clínicos. Dicho de otro modo: eliminar los estresores que desencadenan el burnout, mejorando la calidad de vida y la cultura organizacional y dotar de estrategias de afrontamiento a las personas con mayor vulnerabilidad de sufrir estrés. Phillips (1984) incide en la necesidad de conocer todas las variables implicadas en el síndrome de quemarse a la hora de establecer un programa preventivo. Según este autor, la intervención debe contemplar tanto los procesos cognitivos de evaluación de los sujetos y el desarrollo de estrategias cognitivo-conductuales que les permiten mitigar la fuente de estrés, como la adquisición y fortalecimiento de las habilidades sociales y del apoyo social, así como intentar eliminar o disminuir los estresores del entorno organizacional que dan lugar al desarrollo del síndrome. Los programas de intervención pueden distinguirse, pues, según su objetivo, ya sea sobre el control de estresores, o sobre los procesos de evaluación o estrategias de afrontamiento, y según el nivel en el que se centra la intervención: individual, organizacional o en el nivel individuoorganización (Gil-Monte y Peiró, 1997).

Las estrategias individuales tienen sentido si consideramos que el síndrome de quemarse por el trabajo es un proceso cognitivo mediante el cual el sujeto evalúa y se enfrenta a una experiencia de estrés o convive con el síndrome. En este proceso, el afrontamiento sería el esfuerzo cognitivo y conductual que se desarrolla para manejar las demandas específicas externas $y / 0$ internas que son evaluadas como excedentes o desbordan- tes de los recursos del individuo (Lazarus y Folkman, 1994). Las estrategias de afrontamiento pueden estar dirigidas a regular la respuesta emocional (disminuir el grado de trastorno emociónal que genera la situación estresante), o dirigidas a manipular el problema. Las estrategias de afrontamiento de control previenen el síndrome de burnout porque aumentan los sentimientos de realización personal en el trabajo, mientras que las de escape facilitan la aparición del burnout (Ceslowitz, 1989; Leiter, 1990; 1991). Tanto el uso de estrategias centradas en el problema, como las dirigidas a la emoción, predicen una significativa proporción de cambio en los niveles del síndrome (Boyle, Grap, Younger y Thornby, 1991). Según algunos estudios, las formas de afrontamiento pueden tener efectos directos e indirectos sobre el síndrome de quemarse en el trabajo (Boyle y col., 1991; Leiter, 1990, 1991); sin embargo, para Koeske, Kirk y Koeske (1993), estas estrategias tendrían una relación más moduladora que directa entre el estrés laboral y el síndrome de burnout.

Matteson e Ivancevich (1987) clasifican los programas de adquisición de destrezas de afrontamiento del estrés en programas dirigidos a las estrategias instrumentales, cuando tienen como objetivo la adquisición de destrezas para la resolución de problemas, y programas dirigidos a las estrategias de carácter paliativo, cuando su objetivo es la adquisición de destrezas para el manejo de las emociones. Entre las técnicas más empleadas en los programas dirigidos a estrategias instrumentales se encuentran: la resolución de problemas ( $D^{\prime} Z$ Zurilla y Goldfried, 1971), asertividad (Lange y Jakubowski, 1976), manejo de tiempo (Reiner y Hartshorne, 1982), etc. Los programas dirigidos a las estrategias de carácter paliativo suelen ocuparse del entrenamiento y adquisición de habilidades para la expresión de emociones, manejo de sentimientos de 
culpa, relajación, etc. (Cherniss, 1981; Phillips, 1984). La evidencia empírica ha demostrado que son más efectivas las estrategias instrumentales que las de tipo paliativo (Gil-Monte y Peiró, 1996).

Las estrategias que abordan la prevención del síndrome de quemarse en el trabajo desde la perspectiva grupal recomiendan fomentar el apoyo social, la comunicación y las relaciones sociales entre compañeros y supervisores (Carrilio y Eisemberg, 1984; Matteson e Ivancevich, 1987), porque gracias al apoyo social, las personas obtienen nueva información, adquieren nuevas habilidades o mejoran las que poseen, obtienen refuerzo social y retroinformación sobre la ejecución de las tareas y consiguen apoyo emocional, consejos, u otros tipos de ayuda.

Junto con las estrategias citadas anteriormente, cada vez es más usual que los directivos de las organizaciones demanden programas de prevención dirigidos a mejorar el ambiente y el clima de las empresas, ya que los desencadenantes del burnout son estresores crónicos que se dan en el entorno laboral. Variables como el contenido del puesto (sobrecarga laboral, falta de definición en las de tareas, escasa información sobre su desempeño, falta de oportunidad para el empleo de habilidades adquiridas, etc.), disfunciones en el desempeño de los roles (ambigüedad y conflicto de rol), el clima laboral (estructura, responsabilidad, participación, normas, recompensas, etc.), suelen ser antecedentes del síndrome que explican un porcentaje significativo de su varianza. Una de las estrategias más utilizadas en la prevención desde el punto de vista organizacional es la implementación de sistemas de evaluación y retroalimentación, programas que eran escasamente utilizados hasta hace muy poco tiempo por temor a que la evaluación fuera percibida de forma negativa por los empleados. Matteson e Ivancevich (1987) creen que es necesario enfatizar la función de la retro- alimentación, poniendo siempre cuidado en que no se experimente como un juicio. Otro de los programas utilizados son los procesos de desarrollo organizacional, que buscan mejorar la organización a través de esfuerzos sistemáticos y planificados a largo plazo, centrados en lograr una cultura organizacional más eficaz y colaboradora, y en los procesos sociales y humanos de la organización (Beer, 1976). El énfasis de esta estrategia está en la formación de equipos de trabajo, fomentando un ambiente que propicie el crecimiento personal. Destinados a los nuevos empleados, se vienen utilizando los programas de socialización anticipada, dirigidos a evitar que una entrada inadecuada en la organización pueda favorecer el síndrome, por la discrepancia entre la expectativas y la realidad con la que se encuentra el trabajador. Estos programas pueden prevenir el choque anticipando esta experiencia de una manera más moderada antes de comenzar a trabajar y ayudándoles a analizar la situación, así como dotándoles de estrategias de negociación y solución de conflictos (Matteson e Ivancevich, 1987). Otras estrategias organizacionales recomendadas son el rediseño de la realización de tareas (Maslach, 1982) para clarificar la toma de decisiones, mejorar la supervisión, establecer objetivos claros para los roles profesionales y mejorar las redes de comunicación interpersonal (Aber, 1983; Reiner y Hartshorne, 1982); el facilitar que los trabajadores puedan cambiar de actividades, sobre todo en puestos de atención al público que conllevan mucha demanda emocional (Edelwich y Brodsky, 1980) y establecer objetivos claros para los roles profesionales. Se recomienda, igualmente, que la dirección considere cambios que conlleven mejoras de recompensas (Shinn y Morch, 1983) y aumentar la capacidad de autonomía de los trabajadores (Aber, 1983).

Maslach y Leiter (1999), tras muchos años dedicados a la investigación sobre 
el burnout, llegan a la conclusión de que, aunque las variables personales son cruciales, existen ciertas situaciones laborales que presentan mayor potencial de estrés que otras. La intervención individualizada es necesaria, según ellos, en los casos críticos, pero no soluciona el problema desde su raíz. Aunque suponen un mayor coste, está demostrado que las intervenciones organizacionales son una mejor inversión. Para estos autores, el síndrome de quemarse en el trabajo es un problema más grande de lo que cualquiera pueda afrontar por sí solo. Un individuo puede cambiar su conducta, pero sin ayuda no puede transformar su medio social. Consideran que una intervención sobre la empresa tiene más posibilidades de lograr un cambio efectivo, porque dirige el problema al grupo en lugar de dirigirlo cada vez a un individuo y construye un proceso de apoyo mutuo entre los compañeros. Leiter y Maslach (2000) parten de la premisa de que no se trata sólo de un problema de la persona, sino que es fundamentalmente responsabilidad del medio social en el que se trabaja, cuya estructura y funcionamiento se forma por interacción entre las personas. No es el trabajador aislado, sino el desajuste entre él y su medio laboral lo que resulta crítico. Así, se centran en seis áreas de desajuste sobre las que trabajar: la sobrecarga laboral, las recompensas (no sólo económicas), el grado de control que tiene el trabajador sobre su tarea, el sentimiento de comunidad, la presencia o ausencia de equidad y el conflicto entre los propios valores y los de la empresa. Reducir los desajustes en estas áreas requiere un trabajo en equipo.

\section{EL CHEQUEO PREVENTIVO DE LEITER Y MASLACH (2000)}

Leiter y Maslach (2000) consideran que para afrontar eficazmente el problema del estrés laboral y del burnout es necesario eliminar los desajustes que se producen entre la persona y su puesto laboral, y transformar algunos patrones de relación entre los empleados y entre estos y la dirección (Leiter y Maslach, 2000; Maslach y Leyter, 1999). Colocan el burnout en el extremo de un continuo y definen el extremo opuesto como el compromiso de la persona con su labor y con los objetivos de la organización. Un sentimiento, tal vez ideal, por el cual el trabajador desempeñaría sus funciones, no sólo por la motivación de percibir un salario, sino sobretodo porque estaría comprometido con los fines de esa empresa y porque para él supondría una satisfacción personal realizar bien su tarea. Según este modelo, el sujeto, además de percibir unas recompensas económicas (principal motivación de su trabajo), necesita también obtener retroalimentación de sus compañeros y superiores, sintiéndose alguien competente que desempeña una función útil para su comunidad. Esta concepción implica una visión amplia a largo plazo y una decidida inversión en el presente que puede evitar muchas pérdidas y costes mayores en el futuro.

El modelo que ofrecen puede ser aplicado a cualquier organización que muestre un claro respaldo por parte de la dirección y una participación de la mayoría de los trabajadores para mantener el proceso en marcha hasta el final. El procedimiento de chequeo organizacional pretende analizar las áreas de desajuste trabajo/persona en la empresa, conocer no sólo los problemas, sino también los aspectos positivos sobre los que podrá apoyarse una futura intervención frente al burnout. El objetivo es implicar al personal de todos los niveles de una empresa en el reto de desarrollar nuevas ideas y nuevos procesos para resolver los problemas más eficazmente. Todo el proceso comienza con una encuesta a la plantilla sobre aspectos clave de la vida 
organizacional, igual que un auditor operaría para ayudar a la organización a afrontar sus responsabilidades fiscales. El propósito es obtener información para saber hacia dónde se quiere ir, de modo que la organización pueda usar sus recursos para dirigir los temas importantes de forma eficaz. A la hora de interpretar los resultados de la encuesta, es necesario atender especialmente a los niveles de burnout y compromiso, a la magnitud de los desajustes entre la persona y su trabajo (sobrecarga, control, recompensas, sentimiento de comunidad, equidad y conflicto de valores) y a las relaciones entre las estructuras y procesos de dirección con estas seis áreas de desajuste. Una vez procesados y analizados los datos de la encuesta, y con el informe resultante, se está en posición de discutir, tanto con los directivos como con los empleados, las posibles acciones a emprender, diseñar con ellos y llevar adelante el cambio, centrando su intervención en los problemas identificados en el informe.

Exceptuando el modelo de encuesta, el programa para procesar los datos de la misma, y algunos materiales de ayuda, el chequeo preventivo de Leiter y Maslach no incluye más herramientas para implementar el cambio en una empresa dada. A partir de aquí, los instrumentos con los que cuentan los profesionales a la hora de producir un cambio son: la discusión creativa para desarrollar ideas que redirijan las áreas conflictivas identificadas, la resolución de problemas para diseñar un plan que sea reconocido y aceptado por la plantilla y la dirección, un calendario que permita controlar el proceso y los resultados, así como la nueva información que vaya surgiendo y que ayudará a reconducir ciertos aspectos del plan, incluso a reconsiderarlo. Al igual que en un chequeo médico, un chequeo en la organización nos informa de qué áreas presentan problemas y dónde se locali- zan, pero no existe una fórmula a medida para un problema concreto y en cada organización serán necesarias distintas estrategias para abordarlos.

En esta segunda fase, tras interpretar y discutir los resultados del informe, un equipo de cambio, que incluiría a directivos y trabajadores, establecerá un plan de acción que sea ampliamente aceptado y que tendrá que ser evaluado pasado un tiempo desde su implementación. La organización necesita considerar las distintas visiones que tiene la plantilla sobre las seis áreas de posible desajuste entre la persona y su trabajo y sobre las estructuras y procesos relevantes de la administración. Para conocer los temas candentes de la plantilla y poder emprender acciones ante problemas críticos, es necesaria en todo momento una óptima comunicación, de modo que nadie se vea frenado a la hora de expresar sus puntos de vista ante la dirección. Por ello, debe respetarse al máximo el anonimato de las respuestas al sondeo. La intervención puede centrarse en una sola área de la vida organizacional o abarcar un conjunto de ellas. Los autores recomiendan no intervenir sobre todas a la vez, ya que la intervención sobre un aspecto suele repercutir beneficiosamente sobre los otros. En cada caso, se discutirán y se decidirán las estrategias que se consideren más adecuadas para resolver los desajustes entre las personas y su medio laboral. Para que el proceso no se detenga, advierten de la necesidad de que toda la plantilla se sienta implicada en él y de que se haya alcanzado un consenso sobre el área o áreas de desajuste en las que se va a actuar primero, y priorizar las acciones que se tomarán para dirigirlo. El proceso de intervención organizacional necesita un proyecto inclusivo y compartido, en el que cada miembro del equipo tenga un papel significativo. Debe tenerse muy presente en todo momento que se trata de una intervención a largo plazo, que sólo puede 
tener éxito si parte de un firme compromiso de la dirección con el bienestar de sus trabajadores y un compromiso de estos con el proceso de cambio en las áreas que se han marcado.

Cualesquiera que sean las soluciones propuestas, no pueden ser implantadas en el vacío, sino dentro del contexto organizacional. Aunque se explica que no existen fórmulas previas para enfrentarse a cada área de desajuste, los autores ofrecen descripciones detalladas de distintas intervenciones en diferentes organizaciones, en las que se ha actuado sobre problemas de sobrecarga laboral en una sección concreta, sobre el conflicto entre los valores de la empresa y los del trabajador, o sobre la falta de control o de equidad, por ejemplo. En todos los casos, la vía para enfrentarse al burnout y alentar un compromiso efectivo con el trabajo es la de redirigir cualquiera de estas seis áreas de desajuste que se hayan identificado como fuente de riesgo. Como se ha dicho, las seis áreas de posible desajuste están interrelacionadas, de modo que la acción en cualquiera de ellas tiende a mejorar al menos a alguna de las demás. Si en este proceso se produce una buena colaboración entre los individuos y grupos, se incrementará el conocimiento mutuo de la gente, resaltándose el sentimiento de comunidad, lo que influirá también de manera positiva en el resto de las áreas.

Los resultados nunca son un fin en sí mismos, sino que deben ser tomados como un proceso en marcha para seguir transformando con éxito los desajustes en el trabajo. Si el equipo de cambio se había dirigido inicialmente a una o dos áreas sobre las que trabajar, podrá continuar, según las conclusiones que se obtengan en la evaluación, tratando de transformar los desajustes en otras áreas. La característica más definitoria del desarrollo del chequeo y de la intervención es que constituyen procesos a largo plazo y su éxito depende de lograr que sean en todo momento muy participativos. Los cambios llevan tiempo, particularmente cuando se trata de un problema crónico y a largo plazo como el caso de este síndrome. Por ello, los autores insisten en que las herramientas básicas deben ser: el compromiso por parte de todos los miembros de la empresa, altas dosis de perseverancia y ceñirse al proceso hasta que se produzca algún avance. Advierten también que la implementación de los cambios es lo más difícil porque las expectativas de éxito no suelen ser realistas. Las intervenciones organizacionales llevan su tiempo y el objetivo debe ser más ambicioso que el de reencauzar una fuente específica de tensión o preocupación. El objetivo es transformar las prácticas y estructuras que influyen en la vida laboral y esto no sólo es difícil de implementar sino también de evaluar. Por todo ello, en el plan de intervención se deben tener en cuenta en todo momento mecanismos para que no se detenga el proceso, tanto en la fase de transición, como cuando se considere que ya se ha alcanzado el éxito en alguna de las áreas.

Cualquier empresa que ponga en marcha este proceso necesitará una evaluación, pasado el tiempo, para comprobar si tuvo éxito y efectuar posibles ajustes. Esto aumentará la credibilidad de la organización hacia sus trabajadores a la hora de plantear una mejora de la vida laboral. Con el primer chequeo como referencia, la organización tiene capacidad para anticipar objetivos razonables de cambio. El seguimiento y la evaluación proporcionan datos comparativos y requieren mucho menos esfuerzo que el chequeo inicial para su puesta a punto, ya que los equipos han desarrollado las habilidades, perspectivas y capacidad necesarias, y para el resto de la empresa es algo que ya resulta familiar. La evaluación debería producirse, según Leiter y Maslach (2000), entre doce y treinta meses des- 
pués del chequeo inicial. Si se realiza mucho más tarde de ese tiempo pueden producirse acontecimientos que disipen el impacto inicial de la intervención. Una vez revisadas las comparaciones entre el chequeo inicial o pretest y el postest, será necesario redactar un informe en el que el tema principal sea si se han producido, o no, los cambios previstos en las áreas que resultaron más conflictivas en el primer chequeo, especificando qué acciones se emprendieron para enfrentarlas y cómo se van a mantener o mejorar de aquí en adelante.

\section{COMENTARIOS AL CHEQUEO PREVENTIVO DE LEITER Y MASLACH (2000)}

Tanto el sentido común, como la evidencia empírica, hacen buena la máxima de "más vale prevenir...». No es necesario, ni productivo, esperar a que el problema se agrave, es más fácil atajarlo cuando todavía es incipiente. Igualmente, es preferible destinar recursos a la investigación encaminada a evitar el burnout, que afrontar los costes que conlleva. Pero como bien señalan Leiter y Maslach, reducir la posibilidad de burnout es sólo una parte de una aproximación preventiva; más importante resulta promover cambios para que las personas encuentren un significado a su trabajo y se sientan implicadas con él. Perseguir la excelencia no debería ser simplemente una máxima publicitaria, la responsabilidad con la calidad es necesaria en todos los aspectos de cada organización. Yendo a la verdadera raíz del problema, la percepción del trabajo como un mero medio para obtener dinero resulta, a la larga alienante, por lo que se hace necesario reestablecer un nuevo sentido de compromiso con las responsabilidades laborales, de modo que el trabajador obtenga una satisfacción por su esfuerzo y logre un sentimiento de pertenencia a una comunidad, a un proyecto común, una percepción de que tiene control sobre sus acciones y que todo ello no esté reñido con sus propios valores. Tales aspiraciones, advierten los autores, son muy difíciles de alcanzar.

Es muy probable que los tratamientos destinados a dotar al individuo de estrategias para afrontar el estrés nunca puedan solucionar el problema en toda su dimensión. El síndrome de quemarse en el trabajo se gesta tanto por las condiciones objetivas como por los tipos de relación que se crean entre las diferentes estructuras de una organización. Por eso, un trabajador que se viera afectado por este problema, no podría iniciar en solitario un proceso de intervención y esperar demasiada cooperación de sus colegas y de sus jefes para dirigir los desajustes persona-trabajo. Tampoco se puede perder la perspectiva de que la persona está inmersa en un medio, de que ese medio laboral está formado por personas, muchas de ellas en distintos grados de crisis, y que en todo momento se están produciendo interacciones entre éstas y su entorno. Todos estos aspectos son tenidos en cuenta en el procedimiento de chequeo organizacional de Leiter y Maslach, al considerar una intervención de tipo global, sin desatender los aspectos de comunicación y participación que hacen fuertes a la colectividad y también a los individuos. Son objetivos del programa: la mejora del ambiente laboral (la sobrecarga, la ambigüedad de funciones, la retroalimentación sobre el desempeño de tareas, etc.), mejorar el funcionamiento de la comunicación entre las estructuras organizacionales, incrementar la responsabilidad y la participación, clarificar y aumentar las recompensas implícitas, etc. En suma, los mismos objetivos que suelen perseguir los programas de tipo grupal para enfrentarse al estrés laboral y que buscan lograr una cultura organiza- 
cional más eficaz y colaborativa, fomentando un ambiente que propicie el crecimiento personal y aumente la capacidad de autonomía de los trabajadores.

Desde esta perspectiva de cambio a largo plazo, Leiter y Maslach (2000) se centran en los niveles organizacional y grupal, considerando que no son las características individuales las que constituyen un factor de riesgo de burnout, sino las propias estructuras y procesos que forman la vida en una organización, por interacción entre la persona y su medio laboral. Aunque los autores, en los manuales que acompañan al tratamiento, se refieren a sus éxitos en múltiples intervenciones organizacionales llevadas a cabo en diferentes empresas de los Estados Unidos, incluyendo varios estudios de casos, no ofrecen ningún dato empírico comparando su eficacia con otros tipos programas preventivos. Por otro lado, manifiestan un excesivo énfasis en cargar la responsabilidad del burnout a la empresa y al tipo de relaciones que se establecen entre sus estructuras, olvidando que, como casi todas las cuestiones en psicología humana y social, suelen ser un asunto de interacción entre múltiples factores, como hemos señalado anteriormente. Si bien es cierto que una gran proporción del problema podría ser resuelta cambiando las condiciones objetivas que generan estrés y mejorando las estructuras y procesos de comunicación, no podemos olvidar que nunca se conseguirá minimizar absolutamente el riesgo de burnout y que cada persona reacciona de formas muy distintas ante las situaciones de estrés, por lo que no debería desatenderse la responsabilidad de dotar a los trabajadores de estrategias de tipo personal para un buen afrontamiento del burnout.

Este programa preventivo, aunque prometedor, plantea algunas objeciones. Por un lado, las enormes dificultades que conlleva su implementación efectiva en la empresa, para que no quede reducido sólo a buenas intenciones o a la simple declaración de principios. Por otro, el punto ya mencionado de la ausencia de apoyo empírico sobre su eficacia. Creemos que es necesaria más investigación sobre la eficacia de este programa de intervención comparada con la de otros tratamientos centrados en la persona, o incluso con la de tratamientos mixtos (organizacionales y personales), donde se evalúen los cambios producidos en la empresa, los niveles de burnout y estrés, así como indicadores objetivos del grado de salud de los trabajadores y su reflejo en las incidencias laborales, como el absentismo, las bajas, o los abandonos del puesto; en estudios preferiblemente longitudinales.

\section{REFERENCIAS BIBLIOGRÁFICAS}

Aber, J.L. (1983). Social policy issues in the prevention of burnout: A case study. En B.A. Farber (eds.), Stress and burnout in the human services professions $\left(2^{\text {nd }} \mathrm{Ed}\right.$., pp. 213-226): New York: Pergamon Press.

Beer, M. (1976). The technology of organization development. En M.D. Dunnette, (eds.), Handbook of industrial and organizational psychology (pp. 937-993). Chicago: Rand McNally.

Boyle, A., Grap, M., Younger, J. y Thornby, D. (1991). Personality hardiness, ways of coping, social support and burnout in critical care nurses. Journal of Advanced Nursing, 16, 850-857.

Bronsberg, B., y Vestlund, N. (1997). No te quemes. Barcelona: Cúmulus.

Buceta, J.M. y Bueno, A.M. (1995). Ámbitos de la intervención psicológica para controlar el estrés e incrementar la salud. En J.M. Buceta y A.M. Bueno (eds.), Psicología y salud: control del estrés y trastornos asociados (pp. 25-49). Madrid: Dykinson.

Burke, R.J. (1987). Burnout in police work: An examination of the Cherniss model. Group and organization studies, 12, 174188. 
Buunk, A.P. y Schaufeli, W.B. (1993). Burnout: A perspective from social comparison theory. En W.B. Schaufeli, C. Maslach y T. Marek (eds.), Professional burnout: Recent developments in theory and research (pp. 53-69) London: Taylor \& Francis.

Carrilio, T.E. y Eisemberg, D.M. (1984). Using peer support to prevent worker burnout. Social Casework, 65, 307-310.

Ceslowitz, S.B. (1989). Burnout and coping strategies among hospital staff nurses. Journal of Advanced Nursing, 14, 553-558.

Cherniss, C. (1980). Professional burnout in human service organizations. New York: Praeger.

Cherniss, C. (1981). Preventing burnout: From theory to practice. En J.W. Jones (eds.). The burnout syndrome: Current research, theory, interventions. (pp. 172-176). Park Ridge, Illinois: London House Press.

D’Zurilla, T.J. y Goldfried, M.R. (1971). Problem solving and behavior modification. Journal of Abnormal Psychology, 78, 107136.

Edelwich, J. y Brodsky, A. (1980). Burn-out: Stages of desillusionment in the helping professions. New York: Human Sciences Press.

Freudenberger, H.J. (1974). Staff Burn-out. Journal of Social Issues, 30, 159-165.

Gil-Monte, P., y Peiró, J.M. (1996). Desgaste psíquico en el trabajo: el síndrome de quemarse. Madrid: Síntesis.

Golembiewski, R.T, (1982). Organization development interventions. En W.S. Paine (eds.), Job stress and burnout, (pp. 229253). Beverly Hills; CA: Sage.

Golembiewski, R.T. (1986). The epidemiology of progressive Burnout: A primer. Journal of Health and Human Resources Administration, 9, 16-37.

Harrison W.D. (1983). A social competence model of burnout. En B.A Farber (eds.), Stress and burnout in the human services professions. (1985, $2^{a}$ ed., pp. 29-39). New York: Pergamon Press.

Jenkins, S. y Calhoun, J.F. (1991). Teacher stress: Issues and intervention. Psychology in the Schools, 28, 60-70.

Jones, J.W. (1980). The Staff Burnout Scale for Health Professionals (SBS-HP). Park Ridge, IL: London House.
Koeske, G.F., Kirk, S.A. y Koeske, R.D. (1993). Coping with job stress: Which strategies work best? Journal of Occupational and Organizational Psychology, 66, 319-335.

Lange, A.J. y Jakubowski, P. (1976). Responsible assertive behavior. Champaign, IL: Research Press.

Lazarus, R.S. y Folkman, S. (1984). Stress, appraisal and coping. New York: Springer.

Leiter, M.P. (1988). Burnout as a function of communication patterns. A study of multidisciplinary mental health team. Group $\&$ Organization Studies, 13, 111-128.

Leiter, M.P. (1990). The impact of family resources, control coping and skill utilization on the development of burnout: A longitudinal study. Human Relations, 43, 1067-1083.

Leiter, M.P. (1991). Coping patterns as predictors of burnout: The function of control and escapist coping patterns. Journal of Organizational Behaviour, 12, 123-144.

Leiter, M.P. y Schaufeli, W.B. (1996). Consistency of the burnout construct across occupations. Anxiety, Stress and Coping, 9, 229-243.

Leiter, M.P. y Maslach, C. (2000). Preventing burnout and building engagement. San Francisco: Jossey-Bass.

Mallet, K.L. (1991). The relationships between burnout, death anxiety, and social support in hospice and critical care nurses. Ann Arbor, Michigan: University Microfilms Iternational (UMI).

Manzano, G. y Ramos, F. (1999). La despersonalización: clave para identificar el síndrome de Burnout en personal de enfermería. Enfermería Científica, 206, 63-78.

Maslach, C. (1976). Burned-out. Human Behavior, 5, 16-22.

Maslach, C. (1981). Burnout: A social psychological analysis. En J.W. Jones (Ed.), The burnout sindrome. Park Ridge, IL: London House Press.

Maslach, C. (1982). Burnout: the cost of caring. Englewood Cliffs, NJ: Prentice-Hall.

Maslach, C. (1982). Understanding burnout: definitional issues in analysing a complex epifenomenon. En W.S. Paine (Ed.), Job, Stress and burnout. Beverly Hills: Sage.

Maslach, C. (1999). The burn-out syndrome and patient care. En C. Garfield (Ed.), Stress and Survival: The emotional realities of life-threatening illness. St. Louis: Mosby. 
Maslach, C., y Jackson, S.E. (1981). The measurement o experienced Burnout. Journal of Occupational Behavior, 2, 99-113.

Maslach, C. y Jackson, S.E. (1982). Burnout in health professions: A social psychological analysis. En G. Sanders y J. Suls (Eds.), Social Psychology of health and illness. Hillsdale, N.J: Erlbaum.

Maslach, C., Jackson, S.E., y Leiter (1996). Maslach Burnout Inventory ( $3^{\text {rd }}$ ed.). $\mathrm{Pa}-$ lo Alto. CA. Consulting Psychologists Press.

Maslach, C. y Leiter M. (1999). The truth about burnout. San Francisco: Jossey-Bass.

Matteson, M.T. e Ivancevich, J.M. (1987). Controlling work stress. Effective human resource and management strategies. San Francisco: Jossey-Bass.

Olmedo, M. y Santed, M.A. (1999). El estrés laboral. Madrid: Klinik.

Parkes, C. (1998). Estrés, trabajo y salud: características laborales, contexto ocupa- cional y diferencias individuales. En J. Buendía (Ed.), Estrés laboral y salud (pp. 79-117). Madrid: Biblioteca Nueva.

Phillips, J.R. (1984). Faculty burnout. American Journal Nursing, 82, 1525-1526.

Pines, A., Aronson, E. y Kafry D. (1981). Burnout: From tedium to personal growth. New York: Free Press.

Pines, A. y Aronson, E. (1988). Career Burnout: Causes and cures, (2nd Ed.). New York: The Free Press.

Ramos, F. (1999). El síndrome de burnout. Cursos de Postgrado en Psicopatología y Salud. Madrid: Klinik.

Reiner, H.D. y Hartshorne, T.S. (1982). Job burnout and the school psychologist. Psychology in the Schools, 19, 508-512.

Shinn, M. y Morch, H. (1983). A tripartite model of coping with burnout. En B.A. Ferber (Ed.), Stress and burnout in the human services professions ( $2^{\mathrm{a}} \mathrm{ed} ., \mathrm{pp}$. 227-240). New York: Pergamon Press. 